\title{
Le cerveau hormonal
}

Des peptides variés sont émis par le cerveau : changent-ils le comportement? Leur nombre et la diversité de leurs fonctions rendent très complexe l'analyse du cerveau hormonal.

\section{Jean-Didier Vincent \\ Professeur de physiologie à l'uni- versité de Bordeaux-II. \\ Directeur de l'unité de Recherches I76 de l'Inserm.}

Elisabeth Arnauld Chargée de Recherche à l'Inserm.

\section{ADRESSE}

J.-D. Vincent, E. Arnauld: Inserm, Unité 176, domaine de Carreire, rue Camille Saint-Saëns, 33077 Bordeaux Cedex.

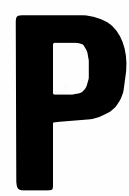

renez un individu normalement constitué et grâce à une fine canule introduite dans son cerveau, saupoudrez une étroite parcelle de son hypothalamus d'une pincée de lulibérine ( $\mathrm{LH} \mathrm{RH})$ : votre cobaye, pour peu qu'il ait une partenaire bien disposée à sa portée, se livrera sur l'heure à une violente et répétitive activité amoureuse. L'histoire est inquiétante, mais soyez rassurés, l'individu en question n'est qu'un rat de laboratoire et il est peu probable qu'Yseult ait usé de lulibérine pour rendre Tristan amoureux: la barque du roi Marc n'était pas un laboratoire de psychophysiologie.

Il n'empêche, le fait expérimental est là. Il suffit de l'injection, dans une région déterminée de l'encéphale, d'une infinitésimale quantité d'un certain peptide pour déclencher chez l'animal la séquence complète de son comportement amoureux depuis les travaux d'approche jusqu'à la consommation de l'acte. Mais l'aventure chimique ne s'arrête pas là. Des dosages ont montré que l'explosion finale du coït s'accompagnait d'une libération massive d'endorphines. Ces peptides seraient responsables par leur action inhibitrice sur les cellules nerveuses de l'hypothalamus, de la satiété sexuelle. Autrement dit, après le peptide du désir, voici assuré, par peptides interposés, le repos du guerrier. Poussons le jeu plus loin. L'injection d'endor- phines dans l'hypothalamus provoque chez le rat un comportement de prise alimentaire. Traduisez : l'amour donne faim! Alors, le désir, l'amour, la faim? La libération en cascade de substances chimiques à l'intérieur du cerveau? Nous ne serons pas assez naifs pour succomber à la tentation d'un réductionnisme si peu romantique.

Que savons nous après tout des peptides sécrétés par le cerveau du jeune Oedipe lorsqu'il fut privé du sein de sa mère... Ce qui nous intéresse, c'est de savoir qu'une substance unique appliquée expérimentalement ou libérée naturellement dans le cerveau est capable de déclencher la séquence complète et ininterrompue des actes moteurs infiniment complexes et variés qui constituent un comportement déterminé. On pourrait multiplier l'exemple avec l'angiotensine [I] et le comportement de boisson, l'ocytocine et le comportement maternel [2], la vasopressine et le marquage du territoire [3]. Ce type d'action inductrice d'un comportement n'est propre ni aux peptides ni aux vertébrés. Kravitz et un groupe de chercheurs de Harvard ont montré que l'injection de sérotonine dans la circulation - à une langouste, déclenche une posture caractéristique du mâle amoureux et en situation de défense: pinces ouvertes, pattes en extension et queue retroussée. Une autre amine - l'octopamine - provoque la pos- 
ture inverse, celle de la soumission et de l'accouplement chez la femelle, pinces fermées, pattes affalées et queue retournée [4].

Nous insisterons sur le caractère stéréotypé de ces comportements qui se répètent identiques à euxmêmes pour chaque espèce. Un tel caractère est, bien sûr, loin d'être évident chez l'homme. Mais plutôt qu'absent, il est masqué, la séquence des actes étant étirée et déformée dans le temps, parfois différée, parfois anormalement prolongée. Il n'est ni de notre propos ni de notre compétence d'analyser les différentes phases du comportement amoureux de l'homme et de la femme. On conviendra tout de même qu'il présente quelques caractères répétitifs et qu'une fois déclenchée, la séquence motrice est souvent irrésistiblement menée jusqu'à son but. La douloureuse cohorte des éjaculateurs précoces est là pour en témoigner.

\section{Les comportements régulateurs}

Un trait caractéristique d'un comportement passionnel, tel que nous le définissons, est le rôle régulateur qu'il joue dans la survie de l'individu ou de l'espèce. Les grands systèmes homéostatiques font tous appel à deux modes d'intervention, l'un comportemental, l'autre métabolique. Pour lutter contre le froid et éviter que la température corporelle ne chute, un animal se met à l'abri, hérisse poils ou plumes (moyens comportementaux) et augmente la production de chaleur par les cellules (moyens métaboliques). Pour compenser une perte d'eau et maintenir constant le degré d'hydratation du corps, l'individu boit (comportement) et freine son élimination d'eau par le rein (métabolisme). Pour maintenir la constance de son poids, il mange (comportement) et mobilise ses réserves (métabolisme), etc.

Le partage qui consisterait à mettre le cerveau - comportement d'un côté, les hormones et glandes - métabolisme - de l'autre n'est pas acceptable. Ce sont souvent les mêmes substances qui interviennent dans les mécanismes de la réponse comportementale et dans ceux de la réponse métabolique, agissant tantôt dans le sang sous la forme d'une hormone, tantôt dans le cerveau sous les espèces d'une neurohumeur. La lulibérine que nous avons vu déclencher dans le cerveau le comportement sexuel règle aussi la maturation des cellules sexuelles et leur éclosion par l'intermédiaire de l'hypophyse. On trouve également de la lulibérine endogène dans l'ovaire où elle agit comme une hormone locale. L'angiotensine qui provoque la vasoconstriction est présente également dans le cerveau où elle déclenche le comportement de boisson, intervient dans la régulation nerveuse de la pression artérielle et stimule la libération de l'hormone antidiurétique. L'ubiquité de cette substance est étonnante puisqu'on la retrouve hormone dans le sang, neurotransmetteur dans les ganglions du système nerveux sympathique, neurotransmetteur encore ou neurohormone enfin aux différents étages du système nerveux central. Même double destinée pour les hormones digestives, insuline, gastrine, peptide intestinal vasoactif (VIP), que l'on retrouve dans le cerveau participant aux mécanismes du comportement alimentaire.

Ainsi, le milieu cérébral est organisé en ensembles fonctionnels qui prennent à leur compte la régulation des grandes fonctions nécessaires à la survie de l'individu et de l'espèce. De la même façon que la formation d'un milieu intérieur chez les êtres pluricellulaires appelle la création d'organes supports de fonctions déterminées, l'apparition d'un milieu cérébral conditionne la formation au sein du cerveau, d'unités régulatrices complexes dont le fonctionnement d'ensemble est assuré par les mêmes entités chimi-

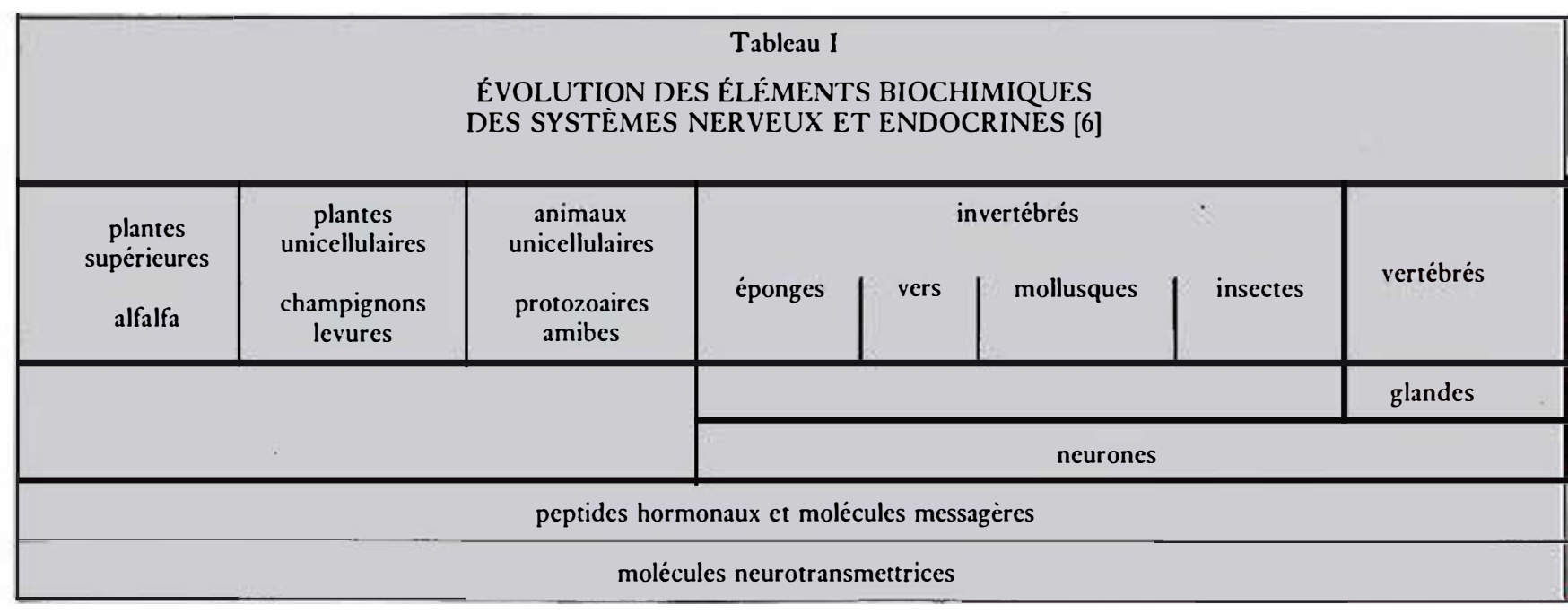


ques que celles utilisées au niveau des organes périphériques. De même que les fonctions homéostasiques du milieu intérieur ne sont pas cantonnées à un organe spécialisé, foie ou rein, les unités homéostasiques du cerveau débordent d'une localisation étroite à des centres comme on dirait un centre de la faim, de la reproduction ou de la respiration - pour s'étendre à des territoires dispersés recouvrant parfois tout l'encéphale. De part et d'autre de la barrière hématoencéphalique, ce sont donc les mêmes agents de liaison qui mènent le double jeu des régulations.

\section{Hormones et évolution}

Les substances chargées de la communication sont présentes dans l'être vivant avant même que ne soient différenciés les appareils. Ce sont d'ailleurs souvent les mêmes substances ou des substances proches, dérivées d'un même gène ancestral que l'on retrouve au long de l'évolution [5]. Hormones et neurotransmetteurs devancent l'apparition des systèmes endocrines et nerveux (tableau I). On pourrait presque dire que les médiateurs précèdent l'apparition de la vie. A ce point de vue, les dérivés de l'adénine et notamment l'adénosine triphosphate (ATP) apparaissent comme les premiers candidats neurotransmetteurs. On peut synthétiser l'adénine en laboratoire en simulant les conditions et la composition de l'atmosphère primitive (eau, ammoniac et méthane) qui régnaient sur la terre avant l'apparition de la vie. Les hormones stéroïdes - œstrogènes et cortisol sont fabriquées par les levures qui possèdent les récepteurs correspondants. Ces mêmes levures synthétisent un peptide de structure très proche de celle de la lulibérine que reconnaissent les récepteurs spécifiques des mammifères. A ce niveau de l'évolution, on observe déjà que ces hormones règlent le développement des cellules, leur reproduction et leurs rapports avec l'hôte qui les abrite. Des hormones ou neuromédiateurs - à ce stade la distinction n'a pas de sens - sont présents chez les protozoaires. On trouve chez Tetrahymena des endorphines, de l'insuline, de la somatostatine. Ces substances sécrétées par l'être unicellulaire peuvent agir à distance sur d'autres individus - action de type phéromone - ou sur la cellule elle-même en se liant à des autorécepteurs - action de type autocrine - toutes actions que nous retrouverons à l'œuvre chez les animaux évolués et chez l'homme (figure I). Un système nerveux et un tube digestif, deux voies de continuité avec le monde extérieur et deux lieux où sont sécrétées les substances chargées de la communication. Chez l'hydre, petit coelentéré d'eau douce, il existe une ébauche avancée de système nerveux (c'est-à-dire de cellules sensibles aux stimuli extérieurs) capable de transporter des informations à travers un réseau de prolongements et de sécréter des neurohumeurs. Parmi celles-ci figurent déjà la dopamine, l'acétylcholine, la séroto- nine et toutes sortes de peptides. Le système nerveux est d'abord neuroendocrine au sens large du terme, les substances sécrétées pouvant agir au voisinage immédiat (synapse) ou à distance de leur lieu d'élaboration. La formation de centres nerveux - rassemblement de neurones et de synapses - chez les vers précède celle de glandes endocrines (tableau I). L'existence de glandes endocrines nettement indépendantes du système nerveux est un avatar relativement récent de l'évolution, propre aux vertébrés et aux invertébrés supérieurs. L'autonomie des systèmes hormonaux nous paraît une condition évolutive déterminante pour le développement ultérieur des centres nerveux.

Sans entrer dans les querelles entre théorie polygénique - origines multiples - et théorie d'une racine commune à toutes les cellules nerveuses, l'évolution nous permet de

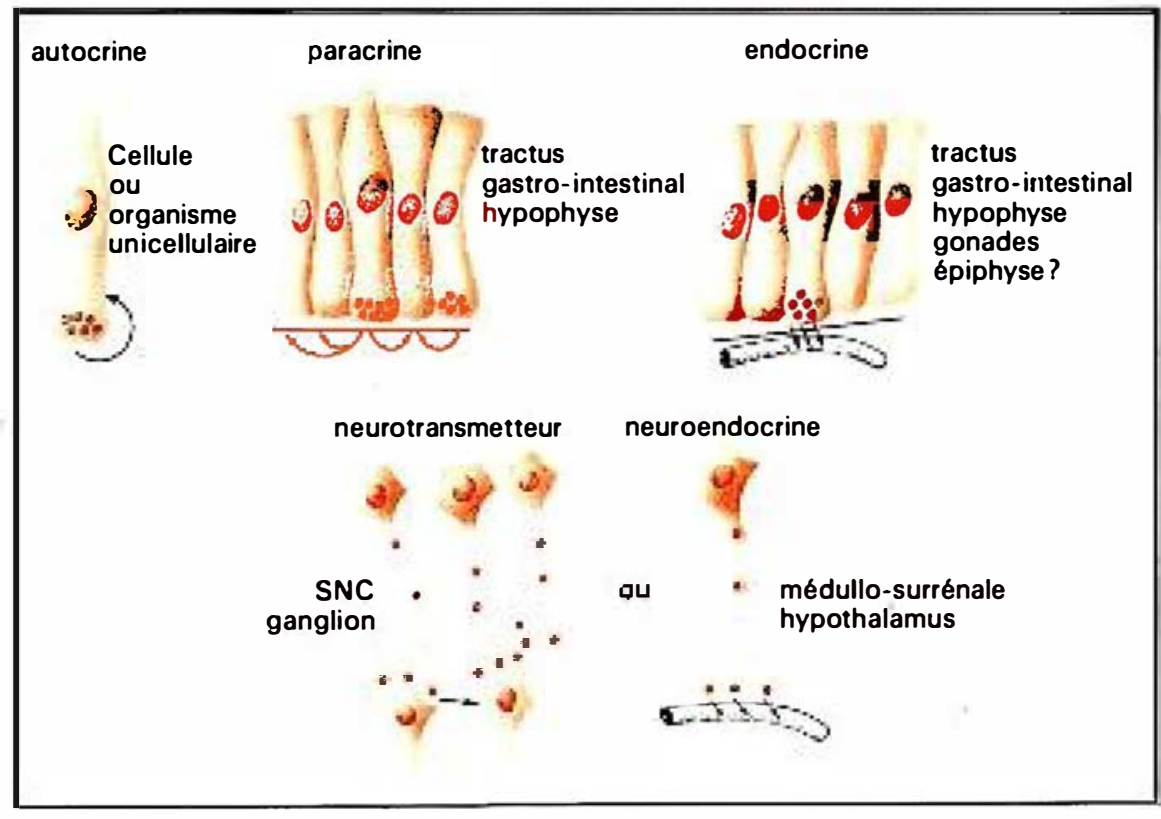

Figure 1. Exemple des modes de sécrétion de peptides. Dans la sécrétion autocrine, le produit sécrété agit localement sur la cellule d'origine. Dans le mode paracrine, le sécrétat exerce une action locale sur les cellules voisines. Dans la sécrétion endocrine, le produit est déversé dans la circulation sanguine pour agir à distance. Les neurones qui communiquent entre eux par des synapses axodendritiques ou axoaxoniques peuvent être considérés comme paracrines (neurotransmetteurs). Les neurones neuroendocrines libèrent leur produits de sécrétion dans la circulation sanguine pour agir à distance [7]. 
comprendre l'extraordinaire diversité et l'ubiquité des neurohumeurs dans les systèmes nerveux, digestif et endocrine.

\section{Du simple au complexe}

Comme le verbe aimer sur une page blanche. Au commencement il y a un gène qui code pour la fabrication d'une première molécule chargée d'assurer la communication pour le compte d'une fonction déterminée (alimentation, défense, reproduction, etc.). Les espèces se différencient, se compliquent; du gène ancestral descendent de nouveaux gènes qui codent pour des molécules nouvelles agissant au sein d'une fonction de plus en plus complexe. Issues d'un même rayon de la bibliothèque génomique, toutes les molécules participant à la fonction auront donc un air de famille, un radical commun qui permet de les reconnaître. A côté d'aimer, il y aura aimable, amour, amical, inamical, amant, amateur; famille de mots unis autour d'une racine commune mais chargés d'un message différent. Parallèlement, les peptides codés par une famille de gènes auront un rôle différent au sein d'une même fonction. Tous les bricolages que la nature peut faire sur un gène substituant un nucléotide par-ci, le dédoublant par-là, le supprimant ailleurs, sont laissés à l'imagination du lecteur et à la science des biologistes moléculaires. L'exemple de l'aplysie illustre ces mécanismes [8]. Dans son cycle de vie annuel, il vient une époque où l'aplysie, animal hermaphrodite, pond des œufs qu'elle dépose par millions sur un support approprié. Les œufs s'échappent de l'orifice génital situé près de la tête grâce à des mouvements de va-et-vient de cette dernière et sont disposés en tas irréguliers. Ce comportement stéréotypé très précis est associé à un arrêt de l'alimentation, de la locomotion et à une accélération de la pompe respiratoire. L'aplysie nous est précieuse par ses 20000 neurones - bien peu de chose comparés aux millions d'un cerveau humain - tous repérables, dénombrables, colorables, qui programment et dirigent l'exécution de ses comportements. Huit cents cellules nerveuses - les bag cells orchestrent le comportement de ponte. Leur activité électrique augmente considérablement dans la demi-heure qui précède la ponte. Les bouffées de potentiels d'action entraînent la libération des neurohormones qui mettent en jeu à leur tour d'autres neurones chargés de la réalisation du programme. Libérées également dans le milieu circulant, ces substances agissent directement sur les glandes sexuelles. Ce sont donc à la fois des hormones et des neurotransmetteurs, selon une confusion des rôles qui nous est maintenant familière. Un extrait de bag cells injecté dans la circulation d'une aplysie adulte, déclenche le comportement de ponte. De cet extrait on tire l'ELH (Egg Laying Hormone), peptide actif de 36 acides aminés. Des chercheurs de l'Université de Columbia ont cloné l'ADN qui code pour l'hormone ELH. A l'aide de cette sonde, une famille de neuf gènes qui semblent tous impliqués dans le contrôle du comportement de ponte a été identifiée. Pour trois d'entre eux la séquence complète des nucléotides est maintenant connue. Ces gènes sont présents dans toutes les cellules de l'aplysie mais ne s'expriment individuellement que dans des territoires déterminés de l'organisme. Le gène qui code pour l'ELH n'est actif que dans les bag cells. Il contient les instructions pour la synthèse d'un précurseur, dont le découpage ultérieur produit, outre l'ELH, d'autres peptides, libérés simultanément par la cellule, dont les actions s'associent et coopèrent à celles de l'ELH. Les deux autres gènes s'expriment au niveau d'une glande annexée à l'appareil génital, la glande atriale. Ils codent pour deux protéines dont le clivage respectif conduit à deux peptides $\mathrm{A}$ et $B$ qui, injectés chez l'animal, sont capables d'activer les bag cells. Huit cents neurones, une glande, et déjà toute la complexité de l'organisation neuroendocrinienne des animaux supérieurs: précurseurs donnant naissance à une kyrielle de peptides à la fois hormones et neurotransmetteurs aux actions synergiques ou enchaînées et famille de gènes s'exprimant en des lieux diffé- rents où ils codent pour des peptides divers mais participant à la même fonction. Pour un seul ELH, déjà que de peptides associés! et nous ne sommes qu'aux branches basses de l'arbre généalogique. En quel coin de notre corps ou de notre cerveau se cache le peptide ELH? L'aplysie qui sommeille dans notre passé se réveille-t-elle au moment de l'accouplement? A quelle fonction l'ELH, s'il existe encore dans notre organisme, participe-t-il ? Son gène oublié et dégénéré a-t-il cessé de s'exprimer?

A côté de la diversification des gènes, on assiste à une diversification des produits codés par un même gène [9], par exemple la proopiomélanocortine (POMC) est scindée dans le cerveau en neuropeptides qui jouent un rôle de neurotransmetteur. Dans les cellules de l'hypothalamus, elle donne naissance à des neurohormones qui sont libérées dans le système porte hypophysaire. Dans l'hypophyse, elle est à l'origine de l'hormone corticotrope libérée dans la circulation générale. Dans le tube digestif enfin et dans l'appareil reproducteur, les dérivés de la POMC libérés sur place ont une fonction paracrine. La liste de ces gènes codant pour des polyprotéines s'accroît rapidement. La diversité peut s'exprimer au sein d'une même cellule au cours du développement de l'individu. Ainsi, les cellules pancréatiques seraient dopaminergiques pendant une période transitoire avant de sécréter de l'insuline. Diversité également par la présence de plusieurs substances au sein d'une même cellule; localisation dans le même neurone de plusieurs neurotransmetteurs, peptide et amine, plusieurs peptides, acétylcholine et sérotonine, acétylcholine et peptide(s), acétylcholine ou amines et purines, etc. Il est bien violé le principe de Dale : à chaque neurone, son neurotransmetteur [1o]. Diversité des fonctions d'une même substance qui peut aussi tenir à l'évolution de ses récepteurs. Les récepteurs de la prolactine situés sur le rein expliquent que cette hormone intervienne chez le poisson dans la régulation de l'eau; cette fonction a disparu chez les mammifêres dont les récepteurs à la prolactine sont situés 
sur le cerveau et sur la glande mammaire. Dans cette confusion des langues, qui peut encore parler de codage de l'information? Remarquons que la présence d'une substance réputée neuromédiatrice dans une cellule ne signifie pas qu'elle serve obligatoirement à une fonction de communication. Que fait, par exemple, l'acétylcholine dans les cellules de la cornée ? Tous les peptides découverts dans une cellule sont-ils fonctionnels?

\section{Le cerveau glande}

Le cerveau est à l'abri de la barrière hémato-encéphalique. Un tel isolement n'a de sens qu'en fonction des voies de communication qui le rompent: entrées par où parviennent les messages nerveux et hormonaux, sorties par où sont ordonnées les actions qui s'exercent alentour. A côté de la sortic nerveuse par les motoneurones, il existe une sortie hormonale au niveau de l'entonnoir étroit formé par le plancher du diencéphale : l'hypothalamus. En ce lieu, le cerveau devient une véritable glande endocrine qui déverse ses produits de sécrétion dans le sang de la circulation générale ou le système porte hypophysaire (figure 2). Les hormones du cerveau obéissent aux deux principes fondamentaux qui définissent une hormone: une action à distance (principe de Hardy) et une autorégulation par rétroaction (principe de Moore et Price).

\section{Les actions à distance}

Nous envisagerons successivement le système magnocellulaire hypothalamique qui déverse ses hormones, ocytocine et vasopressine dans la circulation générale et le système parvocellulaire hypothalamique dont les hormones libérées dans la circulation porte hypophysaire exercent leur action par l'intermédiaire de l'adénohypophyse. La cellule neurosécrétrice magnocellulaire dont les terminaisons se rassemblent dans la post-hypophyse offe l'exemple type d'une cellule peptidergique. Ce modèle a permis notamment l'étude des mécanismes de synthèse, de transport et de libération d'un neuropeptide (hormone ou neurotransmetteur). Le peptide est d'abord synthétisé sous la forme d'un précurseur. Celui-ci mûrit dans des granules au cours de son transport axonal, tandis que les facteurs électriques excitateurs et inhibiteurs, intégrés par la membrane neuronale, sont traduits sous forme de potentiels d'action qui se propagent jusqu'à la terminaison axonale. A ce niveau, le potenticl d'action provoque l'ouverture de canaux membranaires pour l'entrée du calcium. Celui-ci déclenche la rupture des granules à l'extéricur de la terminaison (exocytose) et la libération des peptides qu'ils contiennent. La libération de ces hormones (ocytocine et vasopressine) donne licu à de véritables réflexes dans lesquels les stimuli déclenchent une libération d'hormone au lieu d'une réponse motrice comme c'est le cas dans les réflexes sensitivo-moteurs. L'étude de ces réflexes neurohormonaux a permis de mettre en évidence l'organisation complexe des systèmes neurosécrétcurs et les propriétés particulières des neurones qui les constituent. Signalons seulement que le neurone à vasopressine semble doté d'une activité rythmique interne et que les cellules à ocytocine modifient leur organisation au cours de la lactation. On assiste dans ce dernier cas à un véritable bouleversement de l'architecture des centres nerveux magnocellulaires, lié de façon parfaitement réversible à l'état physiologique dans lequel est momentanément plongé l'animal [ I I]. Ces neurones ont également une intéressante propriété, celle d'être stimulés par le produit de leur propre sécrétion (propriété appclée rétroaction posi-

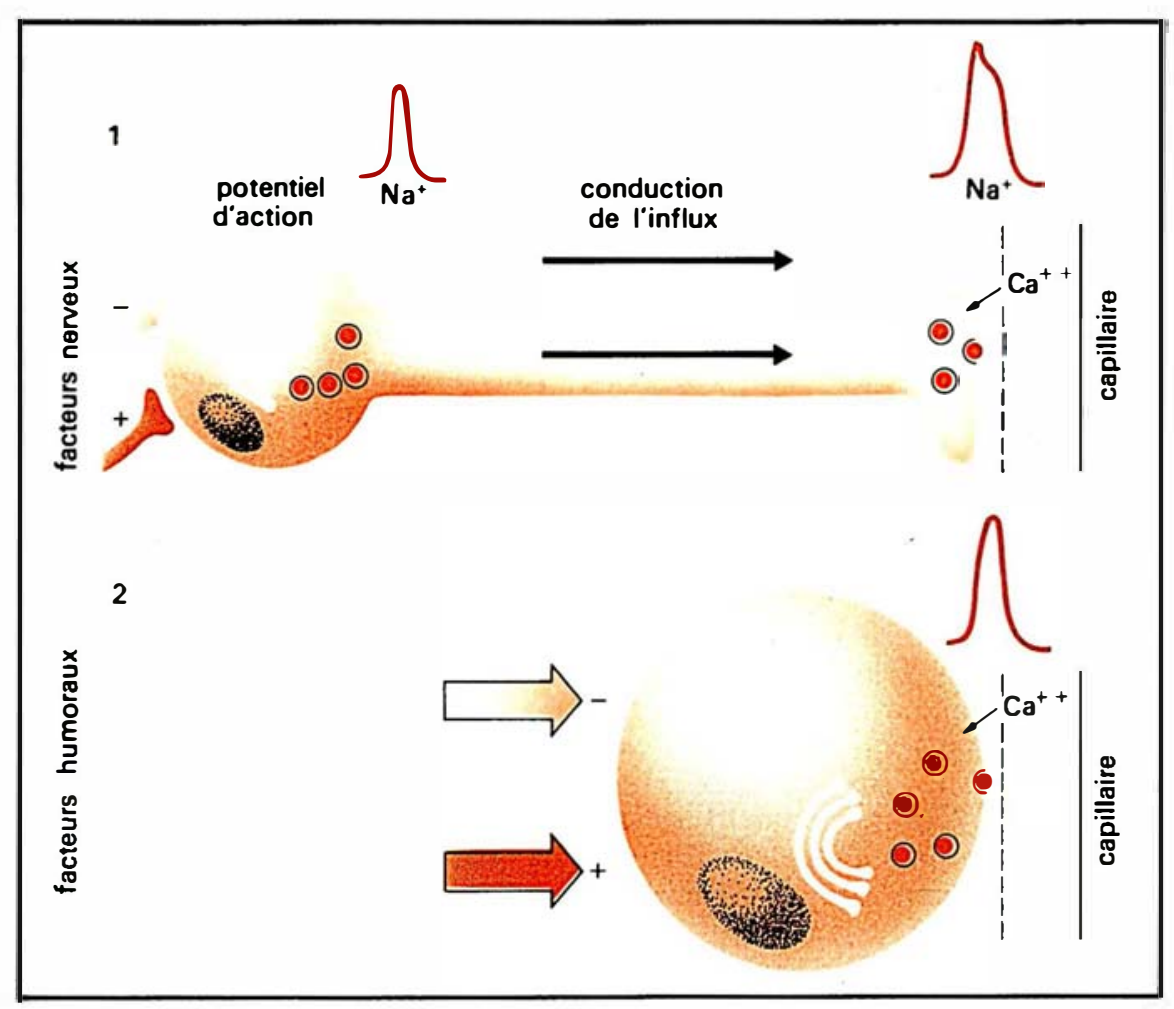

Figure 2. Comparaison entre une cellule neuroendocrine type (1) et une cellule endocrine type (2). Dans les deux cas, le peptide est synthétisé dans le corps cellulaire sous forme d'un propeptide et empaqueté sous forme de granules. Un processus de maturation du propeptide (ou prohormone) se produit pendant le transport axonal pour la cellule neuroendocrine, dans le corps cellulaire pour la cellule endocrine. Dans les deux cas, les stimuli de la sécrétion sont intégrés sous forme de potentiels d'action qui permettent l'entrée de $\mathrm{Ca}^{2+}$ dans la terminaison axonale ou dans la cellule. L'augmentation du $\mathrm{Ca}^{2+}$ interne provoque la libération du produit de sécrétion par exocytose. 
tive ou autoamplification).

Le système parvocellulaire libère un nombre élevé d'hormones au niveau d'une région spécialisée du plancher de l'hypothalamus (voir encadré). Les terminaisons des cellules neurosécrétrices y sont au contact de vaisseaux sanguins qui irriguent l'hypophyse antérieure. Il s'agit soit d'hormones peptidiques qui stimulent (les libérines) ou inhibent (les inhibines) les sécrétions de l'hypophyse antérieure, soit de neurotransmetteurs classiques (GABA, dopamine) qui, libérés dans le sang, acquièrent ici un statut hormonal. Ces hormones exercent leur action sur les différents types de cellules adénohypophysaires. La régulation est toujours multifactorielle, chaque hormone hypophysaire étant sous la dépendance de plusieurs agents, d'origine cérébrale ou autre, et un même agent intervenant dans la régulation de plusieurs hormones. Cette diversité permet un spectre étendu de réponses neuroendocrines qui s'intègrent dans l'ensemble des régulations adaptatives. Ainsi en est-il de l'hormone corticotrope $(\mathrm{ACTH})$ et de l'hormone lactotrope (prolactine), libérées dans toutes sortes de circonstances impliquant une réponse du corps à une situation nouvelle. Ces réponses hormonales sont modulées par l'interaction des multiples facteurs sollicités par le changement du milieu. Il est par ailleurs tout à fait remarquable que la cellule adénohypophysaire rassemble les caractéristiques d'une cellule endocrine stricto sensu et d'une cellule nerveuse. Elle se révèle excitable et le couplage stimulus-sécrétion y met en jeu des mouvements ioniques comparables à ceux que l'on observe sur une terminaison nerveuse.

\section{Les rétroactions hormonales}

Les hormones stéroïdes traversent la barrière hémato-encéphalique et agissent directement sur le fonctionnement des neurones. Cette action s'exerce généralement par l'intermédiaire de récepteurs cytosoliques qui, lorsqu'ils sont liés à l'hormone, sont capables de modifier le fonctionnement du génome et par là d'influencer la synthèse

\section{Hormones sécrétées par l'hypothalamus}

A - Hormones identifiées sur le plan structural: TRH (thyréostimuline); GNRH (gonadostimuline); SRIF (somatostatine); $\mathrm{CRH}$ (cortico-liberine); GHRH (somatocrinine).

B - Hormones reconnues sur le plan fonctionnel mais non identifiées sur le plan structural: PIF (facteur d'inhibition de la prolactine); PRF (facteur de libération de la prolactine).
C - Hormones possibles (non démontré) : MSH IF (facteur d'inhibition de la mélanostimuline); MSH RF (facteur de libération de la mélanostimuline).

D - Hormones dépourvues d'effet primaire: substance $P$; neurotensine; angiotensine; endorphines et enképhalines.

E - Neuromédiateurs à action hormonale: dopamine; GABA; sérotonine. protéique [12]. Il s'ensuit des modifications durables des comportements et des réponses adaptatives aux stimuli ou encore des rétroactions sur la sécrétion des hormones cérébrales. Les exemples de rétroactions hormonales sur le cerveau sont aussi nombreux que les livres et articles qui leur sont consacrés (figure 3). Citons inévitablement l'action complexe des hormones gonadiques (oestradiol, testostérone et progestérone) sur le comportement sexuel. Les corticoïdes interviennent en première ligne dans la défense de l'organisme face aux agressions ou lors de compromis adaptatifs. Leur libération est sous la dépendance de l'hormone hypophysaire corticotrope, l'ACTH elle-même placée sous la tutelle d'un CRH (cortical releasing hormone). Le cerveau est au centre de la réponse surrénalienne et comme tel, il est soumis à la rétroaction des hormones surrénaliennes. L'injection périphérique de dexaméthasone provoque une chute de l'ACTH qui témoigne du freinage exercé par l'hormone surrénalienne sur ses propres centres nerveux de commande. Il est intéressant d'observer que cette rétroaction négative ne se produit plus chez les malades atteints de dépression nerveuse sévère. Les stéroïdes ont aussi sur la cellule nerveuse des actions rapides qui s'exercent directement sur la membrane et sur l'excitabilité électrique des neurones. Leur rôle dans la communication interneuronale ou neurohormonale est mal connu. Les hormones de nature peptidique agissent aussi au niveau du système nerveux central. Le fait que ces hormones ne traversent pas la barrière hémato-encéphalique pose le problème de leur origine. Nous l'avons vu précédemment, la plupart des hormones systémiques sont également synthétisées et libérées au sein du cerveau, qu'il s'agisse d'hormones digestives (gastrine, cholécystokinine, VIP, substance P), d'hormones tissulaires (angiotensine, bradykinine...) ou d'hormones hypophysaires. Le problème de la connaissance des fonctions des hormones peptidiques endocérébrales et de leurs modes d'action se pose avec acuité. Dire que ces hormones peuvent agir comme des neurotransmetteurs ou des modulateurs n'indique pas pourquoi on retrouve les mêmes substances dans le cerveau et dans la circulation périphérique. Le cas de l'angiotensine illustre clairement le problème. Nous avons déjà signalé les effets multiples de cette hormone. L'angiotensine libérée dans le sang chaque fois que le 


\section{REFEERENCES}

I. Fitzsimons JT. The physiology of thirst and sodium appetitc. Cambridge: Cambridge University Press, 1979.

2. Pedersen CA, Ascher JA, Monroe YL, Prange AJ Jr, Oxytocin induces maternal bchavior in virgin female rats. Science 1982; 216 : 648-9.

3. Ferris CF, Albers HE, Wesolowski SM, Goldman BD, Luman SE, Vasopressin injected into the hypothalamus triggers a stereotypic behavior in golden hamsters. Science 1984; 224: 521-4.

4. Kravitz EA, Bcltz BS, Glusman S, et al. Neurohormones and lobsters: biochemistry to behavior. Trends in Neuroscience 1983; 6: 345-9.

5. Krieger DT, Brain peptides: what, where and why. Science 1983; 222: 975-85.

6. Le Roith D, Shilach J, Roth J. Is there an carlier, phylogenetic precursor that is common to both the nervous and endocrine systems? Peptides (Fayetteville) 1982; 3: 21 .

7. Krieger DT. The multiple faces of proopiomelanocortin, a prototype precursor molecule.Clin Res 1983; 31: 342-53.

8. Scheller RH, Jackson JF, MacAllister LB, Schwartz SH, Kandel ER, Axel R. A family of genes that codes for ELH, a neuropeptide eliciting a stereotyped pattern of behavior in Aplysia. Cell 1982; 28: 707-19.

9. Douglass J, Civelli O, Herbert E. Polyprotein gene expression: generation of diversity of neuroendocrine peptides. Annu Rev Biochem 1984; 53: 665-715

10. Lundberg JM, Hokfelt T. Coexistence of peptides and classical neurotransmitters. Trends in Neuroscience 1983; 6: 325-32.

II. Theodosis DT, Poulain DA, Vincent JD Possible morphological bases for synchronisation of neuronal firing in the rat supraoptic nucleus during lactation. Neuroscience 1981; 6: 919-29.

12. McEwen BS, Pfaff DW. Hormone effects on hypothalamic neurons: analysing gene expression and neuromodulator action. Trends in Neuroscience $1985 ; 8$ : 105-10.

13. Reid AI. Action of angiotensin II on the brain: mechanisms and physiologic role. $A m \mathcal{F}$ Physiol 1984; 246: F 533-43.

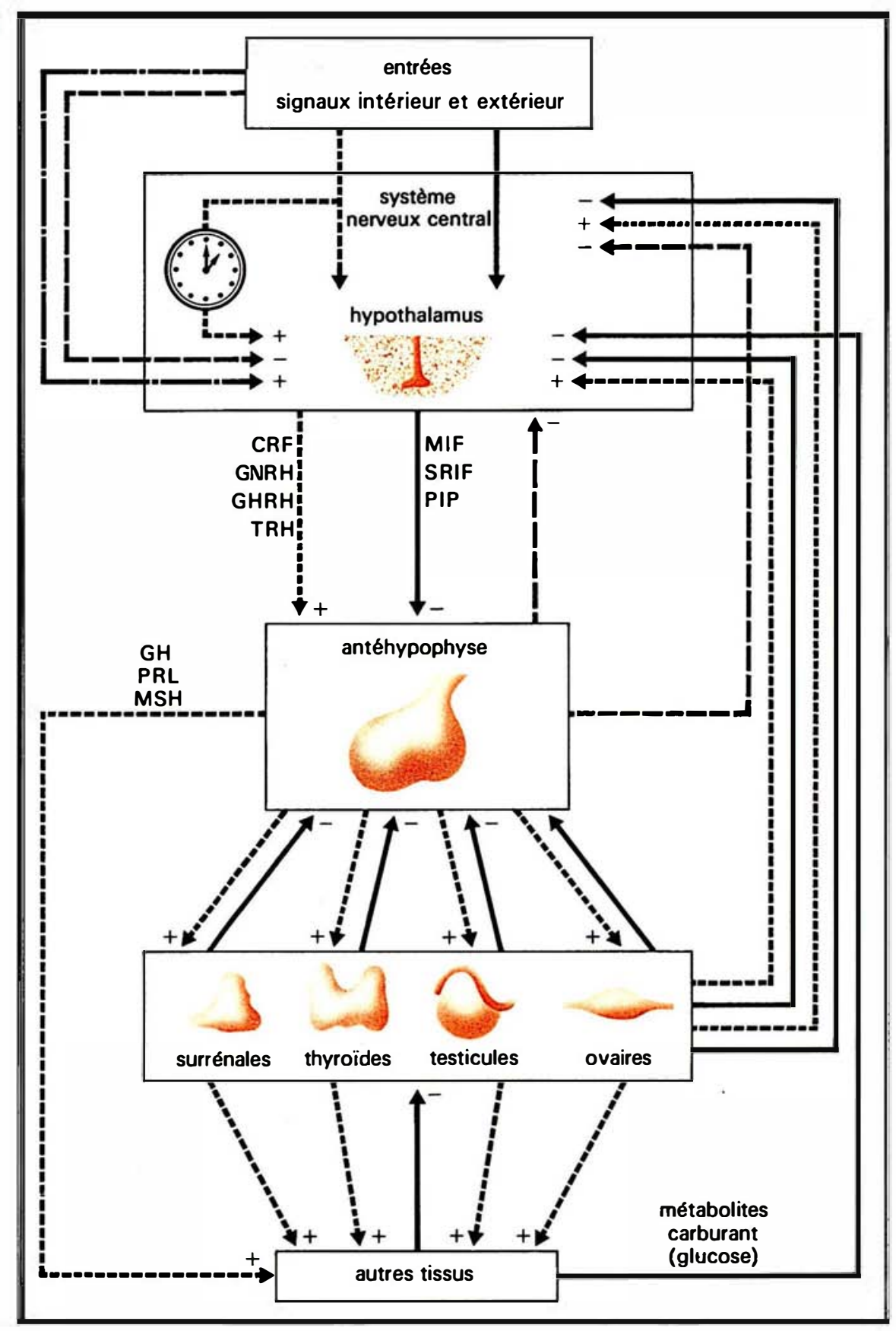

Figure 3. Organisation générale neuroendocrine. 
volume sanguin diminue, agit aussi au niveau du cerveau où nous l'avons vu provoquer le besoin de boire, une élévation de la pression sanguine et une libération de vasopressine, trois effets qui concourent au même résultat, restaurer le volume sanguin. L'angiotensine de la circulation ne traversant pas la barrière hémato-encéphalique, il faut donc admettre qu'il existe dans le cerveau une angiotensine endogène que retrouvent effectivement les dosages et l'immunocytologie et la biologie moléculaire [13]. L'hormone périphérique et la neuro humeur cérébrale participent donc à la même homéostasie et tout se passe comme si une régulation nerveuse centrale utilisant les mêmes agents chimiques venait en quelque sorte doubler, avec la finesse adaptative que lui confère la complexité centrale, la régulation hormonale systémique. La même proposition peut être faite pour la vasopressine. Celle-ci existe au sein du cerveau où des voies contenant de la vasopressine ont été clairement démontrées par l'immunocytologie. Les mêmes stimuli osmotiques et circulatoires qui libèrent la vasopressine dans le sang provoquent sa libération dans le cerveau. On peut donc faire l'hypothèse que la vasopressine centrale participe aux mêmes régulations homéostasiques que la vasopressine systémique, ce qui n'exclut pas sa participation à d'autres fonctions. On se rappellera que ce peptide est un pionnier.

\section{La communication hormonale}

Rappelons d'emblée que la distinction entre neurotransmetteurs et hormones ne répond plus entièrement à la réalité des faits. Sur le plan spatial, des messagers d'origine neuronale agissent à distance de leur cellule d'origine. La substance libérée à l'extrémité neuronale diff use en dehors de l'espace synaptique et s'adresse à des neurones voisins dépourvus de liaisons synaptiques avec le neurone émetteur. Ce cas est observé dans les ganglions sympathiques, où un peptide voisin de la lulibérine présente une action de ce type. Le messager peut également être libéré au niveau d'arbori- sations terminales multiples et très dispersées qui ne présentent pas de différenciation synaptique. $\mathrm{Au}$ niveau du cortex, certains neurones arrosent de leurs messagers une large surface, sans établir de véritable connection avec les neurones qui s'y trouvent. Les informations transmises par de tels messagers concernent de vastes parties du cerveau dont elles réglent le fonctionnement d'ensemble. Elles pourraient être responsables d'une sorte d'homéostasie locale contrôlant le micro-environnement au niveau d'ensembles neuronaux déterminés. On peut imaginer que ces régulations sont liées aux émotions, aux humeurs et à tout ce qui constitue les fonctions "instinctives". Ce cerveau flou, responsable de la part affective et passionnée de l'individu, serait en quelque sorte superposé au cerveau câblé responsable des fonctions sensori-motrices cognitives et rationnelles.

Sur le plan opérationnel, une action neurotransmettrice stricte consiste en l'ouverture passive de canaux ioniques provoquée par un signal électrique dont l'intégration finale permettra au neurone d'émettre à son tour un influx. Certains messagers d'origine nerveuse peuvent avoir une action de type hormonal par intervention d'un deuxième messager qui permet la diffusion de l'information à l'ensemble de la cellule réceptrice et modifie ses propriétés énergétiques ou son excitabilité. On doit saisir encore qu'un même messager de même nature chimique peut parvenir à la membrane réceptrice avec des concentrations différentes selon qu'il est apporté par voie synaptique (forte concentration) ou par voie hormonale (faible concentration); son action sera alors différente. Quant à la cellule, elle possède par ailleurs des récepteurs de sensibilité différente pour la même substance!

\section{Conclusion}

Ces quelques données montrent qu'il existe à côté du cerveau neuronal, exemple d'ordinateur d'une complexité sans modèle, un véritable cerveau hormonal qui modifie sans cesse et dans toutes ses structures le fonctionnement du premier

\section{Summary}

The concept of hormones refers to substances carrying a message at a distance by the blood stream. The brain can thus be considered as an endocrine gland since it releases hypothalamic hormones directly into the blood stream from the neurohypophysis (vasopressin and ocytocin) and the median eminence (releasing factors). Moreover, the secreting neurones display most of the properties of endocrine cells for synthesis, storage and release of their hormones. However, the secretory products are also released within the brain, to act as neuromodulators or neurotransmitters. In the past decade, other peptidergic substances usually considered only as peripheral hormones were shown to be synthetized and released within the brain. Their role is still unclear, but they seem implicated in systems of regulation, for example, angiotensin and blood pressure regulation. Besides the neuronal brain and its complex networks, there is an hormonal brain. The latter can modify and modulate with an infinity of possibilities the functions of the former.

\section{TIRES A PART}

J.-D. Vincent: Inserm, Unité 176, domaine de Carreirc, rue Camille Saint-Saëns, 33077 Bordeaux Cedex. 